\title{
Imaging Red Supergiants with VLT/SPHERE/ZIMPOL
}

\author{
E. Cannon ${ }^{1}$, M. Montargès ${ }^{1}$, L. Decin ${ }^{1}$, A. de Koter ${ }^{1,2}$ \\ ${ }^{1}$ Instituut voor Sterrenkunde, KU Leuven, Leuven, Belgium \\ ${ }^{2}$ Anton Pannekoek Institute of Astronomy, University of Amsterdam, The Netherlands
}

\section{Introduction}

In the red supergiant (RSG) phase of evolution, massive stars show powerful stellar winds which strongly influence the supernova progenitor properties and control the nature of the compact object that is left behind. Here we present VLT/ SPHERE/ZIMPOL data on four RSGs, calibrate the flux and perform a deconvolution using their respective calibrator stars. Comparing these images to models will then allow us to further constrain the mass-loss properties of the RSGs.
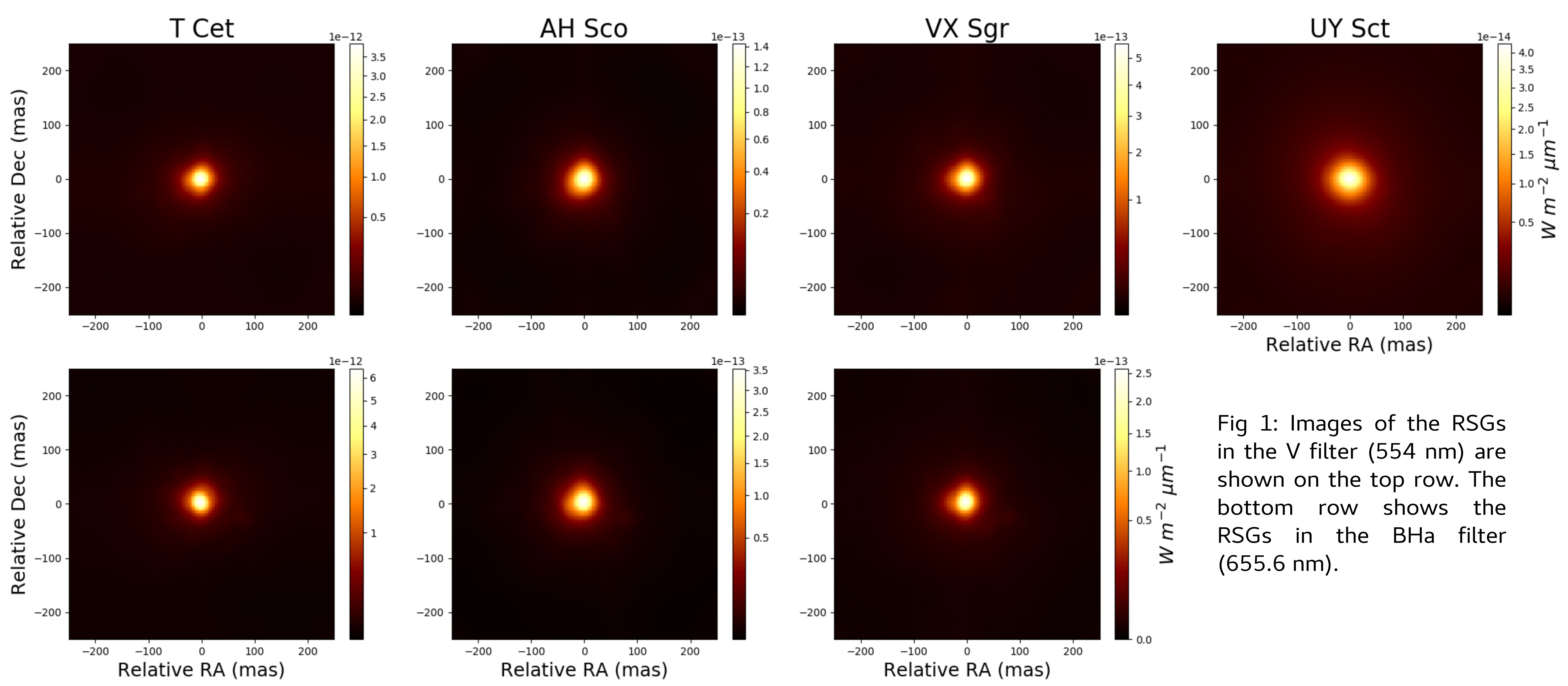

\section{Polarisation}

From the observations, we compute the degree of linear polarisation. This polarisation signal may be caused by anisotropic scattering on dust grains.
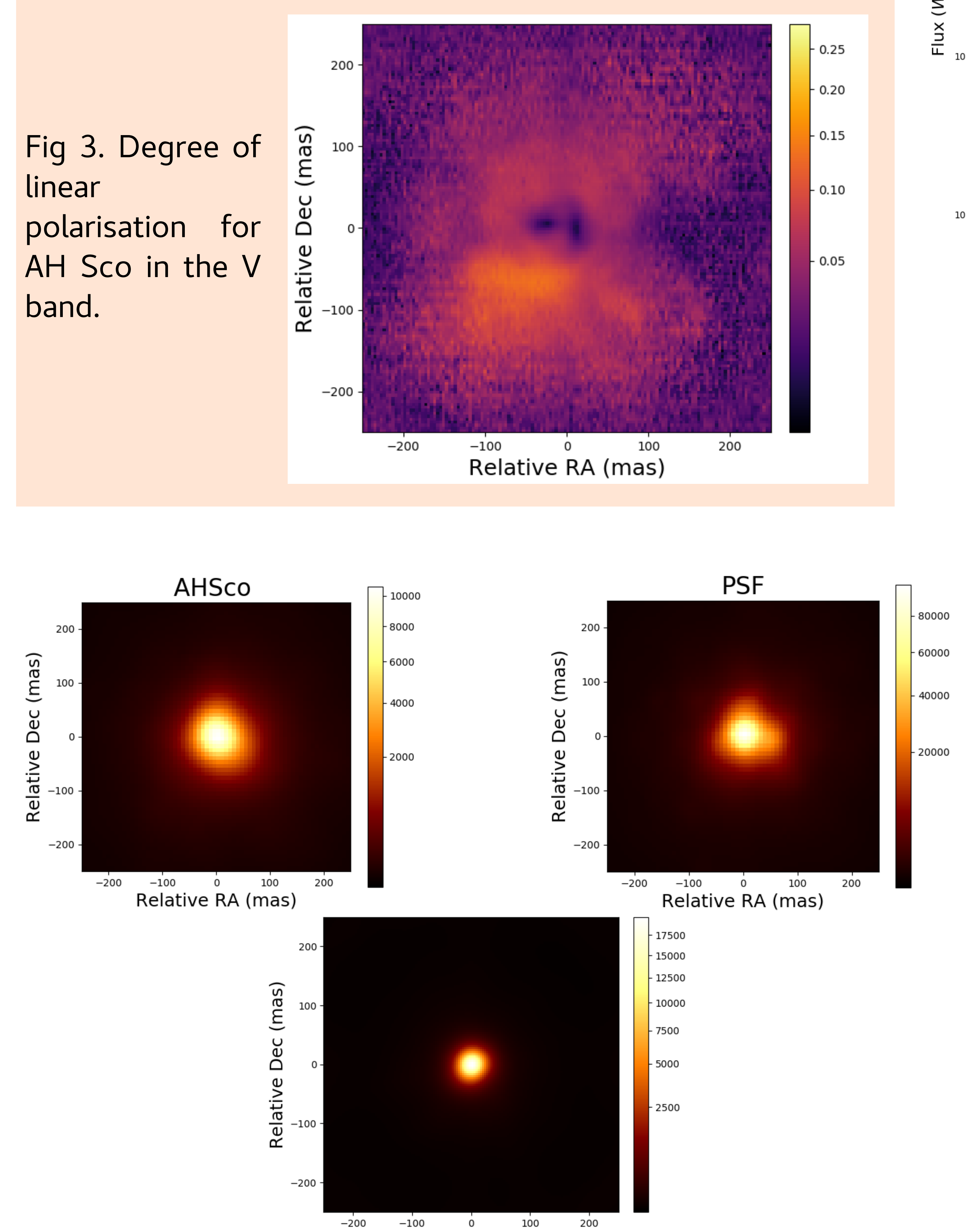

Fig 4: The top row shows images of AH Sco and its PSF. The bottom shows the Lucy-Richardson deconvolved image (4 iterations) of AH Sco. Work on the deconvolution process is ongoing.
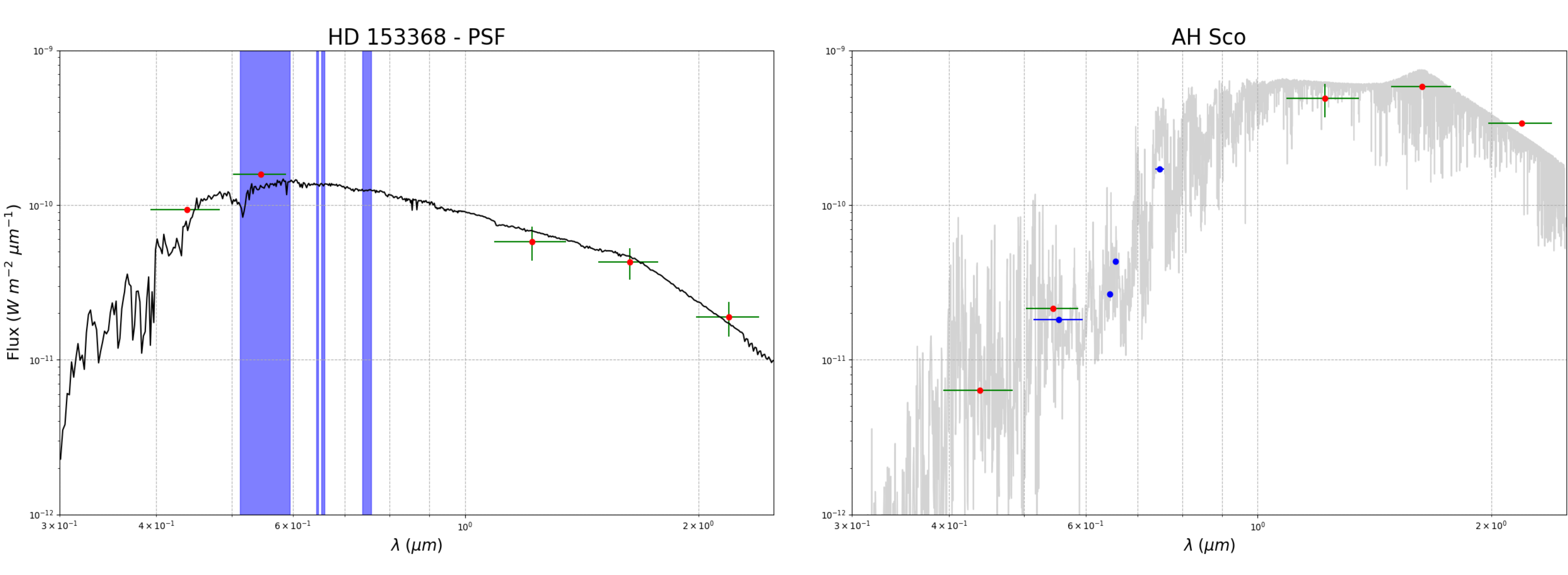

Fig 2: Left: The photometry (taken from literature) of HD153368 (PSF) is plotted in red. The blue bands correspond to the four SPHERE filters observed and the black line to the best matching stellar atmosphere model from the Castelli and Kurucz Atlas ${ }^{[1]}$. Right: The photometry of $\mathrm{AH}$ Sco is shown in red and the derived fluxes from the SPHERE data in blue. A PHOENIX ${ }^{[2]}$ model with $T_{\text {eff }}=3200 \mathrm{~K}$ is shown in grey.

\section{Future Work}

The overall goal of this project is to better constrain the mass-loss rates and parameters of RSGs such as:

- The mass loss rate of the observed RSGs

- The shape of the wind and its correlation with the surface properties of the star

- The role of dust in the mass loss mechanism

- The properties of the dust grains (size, shape and composition)

- The impact of dust on the optical emitted flux

This will be achieved through comparing our observations with theoretical models. 